\title{
Decidability and definability results related to the elementary theory of ordinal multiplication
}

\author{
by
}

\author{
Alexis Bès (Créteil)
}

\begin{abstract}
The elementary theory of $\langle\alpha ; \times\rangle$, where $\alpha$ is an ordinal and $\times$ denotes ordinal multiplication, is decidable if and only if $\alpha<\omega^{\omega}$. Moreover if $\left.\right|_{r}$ and $\left.\right|_{l}$ respectively denote the right- and left-hand divisibility relation, we show that $\operatorname{Th}\left\langle\omega^{\omega^{\xi}} ; \mid{ }_{r}\right\rangle$ and $\operatorname{Th}\left\langle\omega^{\xi} ;\left.\right|_{l}\right\rangle$ are decidable for every ordinal $\xi$. Further related definability results are also presented.
\end{abstract}

1. Introduction. The study of decidability and definability issues related to ordinal theories was initiated by Mostowski and Tarski who proved by means of quantifier-elimination that the class of well-ordered structures has a decidable elementary theory $([13,4]$; see also [16] where the result is obtained as a corollary of Rabin's binary tree theorem).

In the sixties, Büchi ([2], see also [10]) proved that for any ordinal $\alpha$, the weak monadic second-order theory of $\langle\alpha ;<\rangle$ is decidable, from which he deduced decidability of the elementary theory of $\left\langle 2^{\alpha} ;+\right\rangle$, where the ordinal power $2^{\alpha}$ is identified with the set of smaller ordinals and + denotes the graph of ordinal addition restricted to those ordinals (actually the latter result holds for any ordinal in place of $2^{\alpha}$ - see Section 3 ).

In this paper we consider the theory of $\langle\alpha ; \times\rangle$ for an ordinal $\alpha$, where $\times$ stands for (usual) ordinal multiplication. The decidability of $\operatorname{Th}\langle\omega ; \times\rangle$, announced by Skolem in [18], was proved by Mostowski [12] as a direct consequence of his results on direct products of structures and Presburger's decidability result for $\operatorname{Th}\langle\omega ;+\rangle$. Other proofs can be found in [3] and [7]. We prove here that the theory of $\langle\alpha ; \times\rangle$ is decidable if and only if $\alpha<\omega^{\omega}$. The undecidability result is obtained by interpreting the theory of the free monoid with two generators. We also investigate definability of elements in $\langle\alpha ; \times\rangle$.

2000 Mathematics Subject Classification: 03E10, 03B25.

This work was completed while the author was holding a post-doctoral Marie Curie training grant (TMR Program) at University of Mons-Hainaut, Mathematics and Computer Science Department, Mons. 
We next consider weaker theories (in terms of expressibility), namely theories of structures $\left\langle\alpha ;\left.\right|_{r}\right\rangle$ and $\left\langle\alpha ;\left.\right|_{l}\right\rangle$, where $\left.x\right|_{r} y$ (resp. $\left.x\right|_{l} y$ ) means that $x$ is a right-hand (resp. left-hand) divisor of $y$. We show, using the FefermanVaught technique, that $\operatorname{Th}\left\langle\omega^{\omega^{\xi}} ;\left.\right|_{r}\right\rangle$ and $\operatorname{Th}\left\langle\omega^{\xi} ;\left.\right|_{l}\right\rangle$ are decidable for every ordinal $\xi$.

2. Ordinal arithmetic. In this section we recall some useful results on ordinal arithmetic; all of them can be found in Sierpiński's book [17, Chap. XIV].

Proposition 1 (Cantor's normal form for ordinals). Every ordinal $\alpha>0$ can be uniquely written as

$$
\alpha=\omega^{\alpha_{1}} a_{1}+\ldots+\omega^{\alpha_{k}} a_{k}
$$

where $\alpha_{1}, \ldots, \alpha_{k}$ is a decreasing sequence of ordinals, and $0<a_{i}<\omega$.

Throughout the paper, we will use the abbreviation "CNF" for "in Cantor's normal form". The exponent $\alpha_{1}$ is the degree of $\alpha$ and will be denoted by $\operatorname{deg}(\alpha)$.

Proposition 2. Let $\alpha=\omega^{\alpha_{1}} a_{1}+\ldots+\omega^{\alpha_{k}} a_{k}$ and $\beta=\omega^{\beta_{1}} b_{1}+\ldots+\omega^{\beta_{l}} b_{l}$ be two ordinals $>0(C N F)$.

- If $\alpha_{1}<\beta_{1}$ then $\alpha+\beta=\beta$.

- If $\alpha_{1} \geq \beta_{1}$ and $\alpha_{j}=\beta_{1}$ for some $j$, then $\alpha+\beta=\left(\omega^{\alpha_{1}} a_{1}+\ldots+\omega^{\alpha_{j-1}} a_{j-1}\right)+\omega^{\alpha_{j}}\left(a_{j}+b_{1}\right)+\left(\omega^{\beta_{2}} b_{2}+\ldots+\omega^{\beta_{l}} b_{l}\right)$.

- If $\alpha_{1} \geq \beta_{1}$ and $\alpha_{j} \neq \beta_{1}$ for every $j$, then

$$
\alpha+\beta=\left(\omega^{\alpha_{1}} a_{1}+\ldots+\omega^{\alpha_{m}} a_{m}\right)+\left(\omega^{\beta_{1}} b_{1}+\ldots+\omega^{\beta_{l}} b_{l}\right)
$$

where $m$ is the greatest index for which $\alpha_{m}>\beta_{1}$.

In what follows we shall use the following corollary of Proposition 1.

Corollary 3. Every ordinal $\alpha>0$ can be uniquely written as $\alpha=$ $\omega^{\alpha_{0}} \alpha_{1}$ where $\alpha_{1}$ is a successor ordinal.

We say that an ordinal $\alpha>0$ is prime if $\alpha$ cannot be written as the product of two ordinals $<\alpha$; an equivalent definition is that $\alpha$ admits exactly two right-hand divisors. One proves that there are three kinds of prime ordinals: natural prime numbers $<\omega$, ordinals of the form $\omega^{\lambda}+1$ for some $\lambda>0$, and ordinals of the form $\omega^{\omega^{\eta}}$ for some ordinal $\eta$.

The unique factorization theorem for natural numbers no longer holds for ordinals (we have e.g. $(\omega+1) \cdot \omega=\omega \cdot \omega)$; however, if we add conditions on the succession of prime factors we get the following result, due to Jacobsthal $[9]$ : 
Proposition 4. Every ordinal $\alpha>0$ can be written in a unique way as (1)

$$
\alpha=\left(\omega^{\omega_{1}}\right)^{n_{1}} \ldots\left(\omega^{\omega^{\tau_{s}}}\right)^{n_{s}} m_{t}\left(\omega^{\lambda_{t}}+1\right) m_{t-1} \ldots\left(\omega^{\lambda_{2}}+1\right) m_{1}\left(\omega^{\lambda_{1}}+1\right) m_{0}
$$

where $s, t<\omega, \tau_{1}, \ldots, \tau_{s}$ is a decreasing sequence of ordinals, $0<n_{i}<\omega$, $0<m_{i}<\omega$ and $\lambda_{i}>0$.

The prime ordinals $\omega^{\omega^{\tau_{i}}}, \omega^{\lambda_{t}}+1$, and the primes $<\omega$ which divide at least one of the $m_{i}$ will be called prime factors of $\alpha$.

The form (1) can be easily deduced from the normal form of $\alpha$ :

Proposition 5. If $\alpha=\omega^{\alpha_{1}} a_{1}+\ldots+\omega^{\alpha_{k}} a_{k}$ and $\alpha_{k}=\omega^{\tau_{1}} n_{1}+\ldots+\omega^{\tau_{s}} n_{s}$ then

$$
\begin{aligned}
\alpha= & \left(\omega^{\omega^{\tau_{1}}}\right)^{n_{1}} \ldots\left(\omega^{\omega^{\tau_{s}}}\right)^{n_{s}} a_{k}\left(\omega^{\alpha_{k-1}-\alpha_{k}}+1\right) a_{k-1} \\
& \cdot\left(\omega^{\alpha_{k-2}-\alpha_{k-1}}+1\right) a_{k-2} \ldots\left(\omega^{\alpha_{1}-\alpha_{2}}+1\right) a_{1} .
\end{aligned}
$$

The next proposition gives the normal form of $\beta \gamma$ from those of $\beta$ and $\gamma$.

Proposition 6. Let $\beta=\omega^{\beta_{1}} b_{1}+\ldots+\omega^{\beta_{k}} b_{k}$ and $\gamma=\omega^{\gamma_{1}} g_{1}+\ldots+\omega^{\gamma_{l}} g_{l}$ be two ordinals $>0$.

- If $\gamma$ is finite then $\beta \gamma=\omega^{\beta_{1}} b_{1} g_{l}+\omega^{\beta_{2}} b_{2}+\ldots+\omega^{\beta_{k}} b_{k}$.

- If $\gamma$ is a transfinite successor ordinal then

$$
\beta \gamma=\omega^{\beta_{1}+\gamma_{1}} g_{1}+\omega^{\beta_{1}+\gamma_{2}} g_{2}+\ldots+\omega^{\beta_{1}+\gamma_{l-1}} g_{l-1}+\omega^{\beta_{1}} b_{1} g_{l}+\omega^{\beta_{2}} b_{2}+\ldots+\omega^{\beta_{k}} b_{k} .
$$

- Otherwise $\gamma$ is a limit ordinal, and then

$$
\beta \gamma=\omega^{\beta_{1}+\gamma_{1}} g_{1}+\omega^{\beta_{1}+\gamma_{2}} g_{2}+\ldots+\omega^{\beta_{1}+\gamma_{l}} g_{l} .
$$

3. The theory of ordinal multiplication. Let us specify our logical conventions and notations. We work within first-order predicate calculus without equality, and confuse (most of the time) formal symbols and their interpretations. We shall consider structures with domain an ordinal $\alpha$, identified with the set of ordinals $\beta<\alpha$, and predicates that correspond to restrictions to $\alpha$ of relations defined on the class of ordinals, such as + and $\times$, interpreted as the graph of ordinal addition and multiplication, or $\left.x\right|_{r} y$ and $\left.x\right|_{l} y$ which are interpreted as " $x$ is a right-hand divisor of $y$ " and " $x$ is a left-hand divisor of $y$ ", respectively. For simplicity we will use a single symbol for each restriction of a relation, e.g. we simply write $\langle\alpha ; \times\rangle$.

The choice to consider $\times$ as a ternary relation yields slightly more general results, because if $\times$ was considered as a binary function then $\alpha$ must be closed under this function, which holds if and only if $\alpha$ is of the form $\omega^{\omega^{\xi}}$.

Given an $\mathcal{L}$-structure $\langle\alpha ; \mathcal{L}\rangle$, and an $n$-ary relation $R$ over an ordinal $\alpha$, recall that $R$ is elementary definable (briefly: definable) in $\langle\alpha ; \mathcal{L}\rangle$ if there exists an $\mathcal{L}$-formula $\varphi$ with $n$ free variables such that $R=\left\{\left(a_{1}, \ldots, a_{n}\right)\right.$ : $\left.\langle\alpha ; \mathcal{L}\rangle \models \varphi\left(a_{1}, \ldots, a_{n}\right)\right\}$. Given a structure $\mathcal{M}$, we denote by $\operatorname{Df}(\mathcal{M})$ the set of elements definable in $\mathcal{M}$, and by $\operatorname{Th}(\mathcal{M})$ the elementary theory of $\mathcal{M}$. 
Let us explain the remark of our Introduction, that Büchi's decidability result for $\operatorname{Th}\left(2^{\alpha} ;+\right)$ for every ordinal $\alpha$ still holds for any ordinal $\beta>0$ in place of $2^{\alpha}$. Indeed, let $\beta=\omega^{\beta_{1}} b_{1}+\ldots+\omega^{\beta_{n}} b_{n}$ be an ordinal $>0(\mathrm{CNF})$. Proposition 2 allows us to show first that $\operatorname{Th}(\beta ;+)$ reduces to $\operatorname{Th}\left(\prod_{i=1}^{n}\left\langle\omega^{\beta_{i}} b_{i} ;+\right\rangle\right)$ (by induction on $\left.n\right)$, and then that $\operatorname{Th}\left(\omega^{\beta_{i}} b_{i} ;+\right)$ reduces to $\operatorname{Th}\left(\omega^{\beta_{i}} ;+\right)(i=1, \ldots, n)$. Thus it suffices to prove that $\operatorname{Th}\left(\omega^{\gamma} ;+\right)$ is decidable for every ordinal $\gamma$; this is obvious for $\gamma=0$, and for $\gamma>0$ we can use Büchi's result since $\omega^{\gamma}=2^{\omega \gamma}$.

We first recall some of Ehrenfeucht's results [5] on ordinal theories.

Given $\alpha, \beta, \gamma>0$, we say that $\alpha$ and $\beta$ are congruent modulo $\gamma$, and write $\alpha \sim_{\gamma} \beta$ if either $(\alpha=\beta$ and $\alpha<\gamma)$, or $\left(\alpha=\gamma \cdot \alpha^{\prime}+\delta\right.$ and $\beta=\gamma \cdot \beta^{\prime}+\delta$ for some $\left.\alpha^{\prime}, \beta^{\prime}>0, \delta<\gamma\right)$.

We say that an ordinal $\beta$ is definable in the class of $\mathcal{L}$-structures $\{\langle\alpha ; \mathcal{L}\rangle$ : $\alpha$ ordinal $\}$ if there exists an $\mathcal{L}$-formula $\varphi(x)$ such that for every $\alpha>\beta$, $\langle\alpha ; \mathcal{L}\rangle=\varphi(x)$ if and only if $x=\beta$.

Theorem 7 ([5], see also [19]). (i) Let $\alpha, \beta>0$ be two ordinals. If $\alpha$ $\sim_{\omega^{\omega}} \beta$ then $\left\langle\omega^{\omega^{\alpha}} ;+, \times\right\rangle \equiv\left\langle\omega^{\omega^{\beta}} ;+, \times\right\rangle$.

(ii) An ordinal $\beta$ is definable in $\{\langle\alpha ;+\rangle: \alpha$ ordinal $\}$ if and only if $\beta<\omega^{\omega^{\omega}}$.

(iii) An ordinal $\beta$ is definable in $\{\langle\alpha ;+, \times\rangle: \alpha$ ordinal $\}$ if and only if $\beta<\omega^{\omega^{\omega^{\omega}}}$.

Let us now turn to the theory of $\langle\alpha ; \times\rangle$ for an ordinal $\alpha$. As an immediate consequence of Theorem 7(i), we get the following.

Corollary 8. Given two ordinals $\alpha, \beta>0$, if $\alpha \sim_{\omega^{\omega}} \beta$ then $\left\langle\omega^{\omega^{\alpha}} ; \times\right\rangle$ $\equiv\left\langle\omega^{\omega^{\beta}} ; \times\right\rangle$.

Proposition 7(iii) states in particular that for any ordinal $\alpha$, every constant $c<\min \left(\alpha, \omega^{\omega^{\omega^{\omega}}}\right)$ is definable in the structure $\langle\alpha ;+, \times\rangle$. As one could expect, the situation changes if we remove + :

Proposition 9. (i) For every ordinal $\xi$, let $\mathcal{C}_{\xi}$ denote the set of ordinals $<\omega^{\xi}$ of the form $\omega^{\beta_{1}}+\ldots+\omega^{\beta_{k}}$ with $\beta_{1}>\ldots>\beta_{k}$. Then $\operatorname{Df}\left(\omega^{\xi} ; \times\right) \subseteq \mathcal{C}_{\xi}$, and $\operatorname{Df}\left(\omega^{\xi} ; \times\right) \cap \omega^{\omega^{\omega^{\omega}}}=\mathcal{C}_{\xi} \cap \omega^{\omega^{\omega^{\omega}}}$.

(ii) For every ordinal $\alpha \geq \omega$, there exists $c<\omega$ which is not definable in $\langle\alpha ; \times\rangle$.

Proof. (i) First consider an ordinal $\beta<\min \left(\omega^{\xi}, \omega^{\omega^{\omega}}\right)$ such that $\beta \notin \mathcal{C}_{\xi}$. This means that $\beta=\omega^{\beta_{1}} b_{1}+\ldots+\omega^{\beta_{k}} b_{k}$ (CNF) with $b_{i} \geq 2$ for some $i$. Let us show that $\beta$ is not definable in $\left\langle\omega^{\xi} ; \times\right\rangle$. Let $p, q<\omega$ be two distinct primes such that $p \mid b_{i}$ and $q \nmid b_{i}$. Consider $f: \omega^{\xi} \rightarrow \omega^{\xi}$ defined as follows: for every $\gamma=\omega^{\gamma_{1}} g_{1}+\ldots+\omega^{\gamma_{k}} g_{k}<\omega^{\xi}$ (CNF), $f(\gamma)$ is the ordinal obtained 
from $\gamma$ by permuting the $p$ - and $q$-valuation in each $g_{i}$. One checks that $f$ is a permutation of $\omega^{\xi}$, and Proposition 6 yields that $f(x y)=f(x) f(y)$ for all ordinals $x, y<\omega^{\xi}$. Therefore $f$ is an automorphism of the structure $\left\langle\omega^{\xi} ; \times\right\rangle$; however, the very definition of $p$ and $q$ shows that $f(\beta) \neq \beta$, thus $\beta$ is not definable in $\left\langle\omega^{\xi} ; \times\right\rangle$.

Now consider an ordinal $\beta<\min \left(\omega^{\xi}, \omega^{\omega^{\omega \omega}}\right)$ in $\mathcal{C}_{\xi}$. One has $\beta=\omega^{\beta_{1}}+$ $\ldots+\omega^{\beta_{k}}$ with $\beta_{1}>\ldots>\beta_{k}$. Let us prove that $\beta$ is definable in $\left\langle\omega^{\xi} ; \times\right\rangle$. It follows from Proposition 5 that one can write $\beta$ as

$$
\beta=\omega^{\beta_{0}}\left(\omega^{\lambda_{t}}+1\right) \ldots\left(\omega^{\lambda_{2}}+1\right) \cdot\left(\omega^{\lambda_{1}}+1\right)
$$

with all $\lambda_{i}>0$. Therefore it suffices to show that every ordinal of the form $\omega^{\alpha}$ with $\alpha<\min \left(\xi, \omega^{\omega^{\omega}}\right)$, and every ordinal of the form $\omega^{\lambda}+1$ with $1 \leq \lambda<\min \left(\xi, \omega^{\omega^{\omega}}\right)$, are definable in $\left\langle\omega^{\xi} ; \times\right\rangle$.

In order to do this, we shall define in $\left\langle\omega^{\xi} ; \times\right\rangle$ auxiliary relations and constants. Indeed, one first defines $\left.\right|_{r}$ and $\left.\right|_{l}$, the equality relation, then the constants 0 and 1 , and then:

- " $x$ is a prime" (using the fact that $x$ has exactly two right-hand divisors),

- " $x$ is of the form $\omega^{\omega^{\xi}}$ " ( $x$ is a prime with at least three left-hand divisors),

- the constant $\omega$ (the only prime which is both a left-hand and right-hand divisor of all primes $\left.\omega^{\omega^{\xi}}\right)$,

- " $x$ is a power of $\omega$ " (every prime which is a left divisor of $x$ is an $\omega^{\omega^{\xi}}$ ),

- " $x$ is a prime $<\omega$ " (which is equivalent to " $x$ is a prime $\neq \omega$ and $\left.\left.x\right|_{l} \omega^{\prime \prime}\right)$,

- " $x$ is a prime of the form $\omega^{\lambda}+1$ for some $\lambda>0$ " (other kinds of primes were defined above).

Now by Theorem 7 (ii), every ordinal $\alpha<\min \left(\xi, \omega^{\omega^{\omega}}\right)$ is definable in $\langle\xi ;+\rangle$, thus one can define each $\omega^{\alpha}$ using the relation " $x$ is a power of $\omega$ " and $\times$.

If $\xi$ is a limit ordinal then for every $\lambda \operatorname{such}$ that $1 \leq \lambda<\min \left(\xi, \omega^{\omega^{\omega}}\right)$, the ordinals $\omega^{\lambda}$ and $\omega^{\lambda+1}$ are definable in $\left\langle\omega^{\xi} ; \times\right\rangle$, and $\omega^{\bar{\lambda}}+1$ can be defined as the unique ordinal $\gamma$ of the form $\omega^{\lambda^{\prime}}+1$ (for some $\lambda^{\prime}>0$ ) such that $\left.\gamma\right|_{l} \omega^{\lambda+1}$ and $\gamma \nmid_{l} \omega^{\lambda}$.

If $\xi$ is a successor ordinal, say $\xi=\xi^{\prime}+1$, then the same arguments show that every ordinal $\omega^{\lambda}+1$ with $1 \leq \lambda<\min \left(\xi^{\prime}, \omega^{\omega^{\omega}}\right)$ is definable. Moreover if $\xi^{\prime}<\omega^{\omega^{\omega}}$ then $\omega^{\xi^{\prime}}+1$ can be defined as the unique ordinal of the form $\omega^{\lambda^{\prime}}+1$ (for some $\lambda^{\prime}>0$ ) which is not a left-hand divisor of any power of $\omega$.

(ii) Let $\alpha=\omega^{\alpha_{1}} a_{1}+\ldots+\omega^{\alpha_{k}} a_{k}$ (CNF). Consider the mapping $f$ we defined in (i), with $\xi=\alpha_{1}+1$, and with two primes $p, q$ chosen such that 
$\max \left(a_{i}\right)<p<q<\omega$; this condition ensures that the restriction of $f$ to $\alpha$ is one-one. We have $f(p)=q \neq p$ thus $p$ (and $q$ ) is not definable in $\langle\alpha ; \times\rangle$.

From Proposition 9(ii) we deduce the following.

COROLlary 10. For every ordinal $\alpha \geq \omega$, the relations $<$ and + are not definable in $\langle\alpha ; \times\rangle$.

We now state the main result of the section.

THEOREM 11. Given an ordinal $\alpha$, the elementary theory of $\langle\alpha ; \times\rangle$ is decidable if and only if $\alpha<\omega^{\omega}$.

Proof. CASE 1: $\alpha<\omega^{\omega}$. We show that $\operatorname{Th}\langle\alpha ; \times\rangle$ is reducible to $\operatorname{Th}\langle\omega$; $\left.\times,(\underline{n})_{n<w}\right\rangle$ (by " $(\underline{n})_{n<w}$ " we mean that we add a constant symbol $\underline{n}$ for each $n<\omega)$, which was shown to be decidable by Mostowski [12].

For this let us write $\alpha=\omega^{n} a_{n}+\omega^{n-1} a_{n-1}+\ldots+\omega^{0} a_{0}$ with all $a_{i}<\omega$ and $a_{n}>0$. To each $\beta<\alpha$, written $\beta=\omega^{n} b_{n}+\omega^{n-1} b_{n-1}+\ldots+\omega^{0} b_{0}$ $\left(b_{i}<\omega\right)$, we associate the $(n+1)$-tuple of integers $\psi(\beta)=\left(b_{0}, \ldots, b_{n}\right)$. Then we shall prove the following two facts:

(1) There exists a formula $\varphi\left(x_{0}, \ldots, x_{n}\right)$ in the language $\left\{\times,(\underline{n})_{n<w}\right\}$ such that

$$
\left\langle\omega ; \times,(\underline{n})_{n<w}\right\rangle=\varphi\left(b_{0}, \ldots, b_{n}\right)
$$

if and only if $\left(b_{0}, \ldots, b_{n}\right)=\psi(\beta)$ for some $\beta<\alpha$.

(2) There exists a formula $\varphi^{\prime}\left(x_{0}, \ldots, x_{n}, y_{0}, \ldots, y_{n}, z_{0}, \ldots, z_{n}\right)$ in the language $\left\{\times,(\underline{n})_{n<w}\right\}$ such that

$$
\left\langle\omega ; \times,(\underline{n})_{n<w}\right\rangle \models \varphi^{\prime}\left(b_{0}, \ldots, b_{n}, g_{0}, \ldots, g_{n}, d_{0}, \ldots, d_{n}\right)
$$

if and only if $\left(b_{0}, \ldots, b_{n}\right)=\psi(\beta),\left(g_{0}, \ldots, g_{n}\right)=\psi(\gamma)$ and $\left(d_{0}, \ldots, d_{n}\right)$ $=\psi(\delta)$ for some ordinals $\beta, \gamma, \delta<\alpha$ such that $\beta \gamma=\delta$.

These two facts will ensure that to each sentence $\theta$ in the language $\{\times\}$ one can associate in an effective way a sentence $\theta^{*}$ in the language $\left\{\times,(\underline{n})_{n<w}\right\}$ such that $\langle\alpha, \times\rangle=\theta$ if and only if $\left\langle\omega ; \times,(\underline{n})_{n<w}\right\rangle=\theta^{*}$ (this construction is rather classical and will be omitted).

To prove (1) one uses the fact that $\beta<\alpha$ if and only if $\psi(\beta)<_{\text {lex }} \psi(\alpha)$ where $<_{\text {lex }}$ denotes lexicographical order; from this observation one easily gets the required formula $\varphi\left(x_{0}, \ldots, x_{n}\right)$ (using only constants).

For $(2)$, the formula $\varphi^{\prime}$ expresses Proposition 6 in terms of $\left(b_{0}, \ldots, b_{n}\right)$, $\left(g_{0}, \ldots, g_{n}\right)$ and $\left(d_{0}, \ldots, d_{n}\right)$. We let the reader convince himself that this can be done within the language $\left\{\times,(\underline{n})_{n<w}\right\}$; observe that the cases " $\gamma$ finite" " $\gamma$ successor ordinal" and " $\gamma$ limit ordinal" in Proposition 6 can be easily expressed in terms of $\left(g_{0}, \ldots, g_{n}\right)$ using $\times$ and the constant $\underline{0}$, and moreover that the formulas of Proposition 6 show that each $d_{i}$ can be obtained from $\left(b_{0}, \ldots, b_{n}\right)$ and $\left(g_{0}, \ldots, g_{n}\right)$ by shifting and multiplying coefficients. 
CASE 2: $\alpha \geq \omega^{\omega}$. We interpret in $\operatorname{Th}\langle\alpha ; \times\rangle$ the theory of concatenation of words over the alphabet $\Sigma=\{1,2\}$, which was shown to be undecidable by Quine [15]. We denote by $\Sigma^{*}$ the set of finite words over $\Sigma$, by $\Lambda$ the empty word and by $\bullet$ the concatenation operation.

Consider the function $\varphi: \Sigma^{*} \rightarrow \alpha$ which maps every nonempty word $m=m_{0} \bullet m_{1} \bullet \ldots \bullet m_{n}\left(\right.$ with $\left.m_{i} \in \Sigma\right)$ to

$$
\varphi(m)=\left(\omega^{m_{0}}+1\right)\left(\omega^{m_{1}}+1\right) \ldots\left(\omega^{m_{n}}+1\right) .
$$

Moreover set $\varphi(\Lambda)=1$. It follows from Proposition 4 that $\varphi$ is an isomorphism from $\left\langle\Sigma^{*} ; \bullet\right\rangle$ to $\left\langle\varphi\left(\Sigma^{*}\right) ; \times\right\rangle$. Thus we only need to define the set $\varphi\left(\Sigma^{*}\right)$ in $\langle\alpha ; \times\rangle$ (note that $\varphi\left(\Sigma^{*}\right) \subset \alpha$ ).

From the proof of Proposition 9(i) we know that the constants $\omega+1$ and $\omega^{2}+1$ are definable in $\langle\alpha ; \times\rangle$. Now we can define the binary relation " $x$ is a successor ordinal and $y$ is a prime factor of $x$ " (which is equivalent to " $y$ is prime, $\omega$ is not a left-hand divisor of $x$, and there exists $z$ such that $\left.z\right|_{l} x$ and $\left.\left.y\right|_{r} z\right)$, and finally $\varphi\left(\Sigma^{*}\right)$, as the set of successor ordinals admitting only $\omega+1$ and $\omega^{2}+1$ as prime factors.

4. The theories of $\left\langle\omega^{\omega^{\xi}} ;\left.\right|_{r}\right\rangle$ and $\left\langle\omega^{\omega^{\xi}} ;\left.\right|_{l}\right\rangle$. Theorem 11 can be sharpened in the following sense:

THEOREM 12. Given an ordinal $\alpha$, the elementary theory of $\left\langle\alpha ;\left.\right|_{r},\left.\right|_{l}\right\rangle$ is decidable if and only if $\alpha<\omega^{\omega}$.

Proof (sketch). The case $\alpha<\omega^{\omega}$ is obvious, as $\left.\right|_{l}$ and $\left.\right|_{r}$ are definable in $\langle\alpha ; \times\rangle$. The undecidability for $\alpha \geq \omega^{\omega}$ can be proved by interpreting in $\left\langle\alpha ;\left.\right|_{r},\left.\right|_{l}\right\rangle$ the behaviour of a two-counter automaton whose halting problem is undecidable $([11]$, see also [8, Sec. 7.8]). Indeed, consider a sequence of successive configurations of such a machine (we deal with a machine with empty input, and counters with nonnegative values), say

$$
\left(c_{0}, c_{0}^{\prime}, q_{0}\right),\left(c_{1}, c_{1}^{\prime}, q_{1}\right), \ldots,\left(c_{n}, c_{n}^{\prime}, q_{n}\right)
$$

where $\left(c_{i}, c_{i}^{\prime}, q_{i}\right)$ is a triple of nonnegative integers such that $c_{i}, c_{i}^{\prime}$ correspond to the content of the two counters and $q_{i}$ encodes the state of the machine at the $i$ th step. The idea is to encode this sequence by any ordinal of the form

$$
p_{1}^{c_{0}} p_{2}^{c_{0}^{\prime}} p_{3}^{q_{0}}(\omega+1) p_{1}^{c_{1}} p_{2}^{c_{1}^{\prime}} p_{3}^{q_{1}}(\omega+1) \ldots(\omega+1) p_{1}^{c_{n}} p_{2}^{c_{n}^{\prime}} p_{3}^{q_{n}}
$$

where $p_{1}, p_{2}, p_{3}$ denote three distinct primes $<\omega$.

The above result suggests the question whether undecidability could occur even in the presence of a single relation $\left.\right|_{r}$ or $\left.\right|_{l}$. The aim of this section is to show this is not the case: we prove that for every ordinal $\xi, \operatorname{Th}\left\langle\omega^{\omega^{\xi}} ;\left.\right|_{r}\right\rangle$ and $\operatorname{Th}\left\langle\omega^{\xi} ; \mid l_{l}\right\rangle$ are decidable. 
We shall use the notion of generalized weak power of structures, which was introduced by Feferman and Vaught [6, Section 9]. Let us recall some related notations and results.

Let $A, B$ be two nonempty sets, and let $e \in A$. We denote by $S^{+}(B)$ (respectively $S^{*}(B)$ ) the set of finite (resp. finite or cofinite) subsets of $B$. We denote by $A_{e}^{(B)}$ the set of functions $f: B \rightarrow A$ such that $\{b \in B: f(b) \neq e\}$ is finite.

Let $\mathcal{A}=\left\langle A ; \mathcal{R}_{A}, e\right\rangle$ be a structure in the language $\mathcal{L}_{A}=\left\{\mathcal{R}_{A}, e\right\}$ where $\underline{e}$ is interpreted as the constant $e$, and $\mathcal{R}_{A}$ is a set of relations over $A$ ( $\mathcal{A}$ is the "factor structure").

Let $\mathcal{B}=\left\langle S^{*}(B) ; \subseteq\right.$, FIN, $\left.\mathcal{R}_{B}\right\rangle$ be a structure in the language $\mathcal{L}_{B}=$ $\left\{\subseteq\right.$, FIN, $\left.\mathcal{R}_{B}\right\}$ where $\subseteq$ and FIN are interpreted respectively as the inclusion relation and the relation "to be a finite set", and $\mathcal{R}_{B}$ is a set of relations over $S^{*}(B)$ ( $\mathcal{B}$ is the "index structure").

Definition 13 . Let $R$ be a $k$-ary relation over $A_{e}^{(B)}$; we say that $R$ is accessible in $(\mathcal{A}, \mathcal{B})$ if there exist an $\mathcal{L}_{B}$-formula $G\left(X_{1}, \ldots, X_{l}\right)$ and $l$ $\mathcal{L}_{A}$-formulas $F_{1}, \ldots, F_{l}$ with $k$ free variables such that for every $k$-tuple $\left(f_{1}, \ldots, f_{k}\right)$ of elements of $A_{e}^{(B)}, R\left(f_{1}, \ldots, f_{k}\right)$ holds if and only if

$$
\mathcal{B}=G\left(T_{1}, \ldots, T_{l}\right)
$$

where

$$
T_{i}=\left\{x \in B: \mathcal{A} \models F_{i}\left(f_{1}(x), \ldots, f_{k}(x)\right)\right\} \quad \text { for every } i \in\{1, \ldots, l\} .
$$

With the above notations, if $\mathcal{R}$ is a set of relations over $A_{e}^{(B)}$, we say that the structure $\left\langle A_{e}^{(B)} ; \mathcal{R}\right\rangle$ is a generalized weak power of $\mathcal{A}$ relative to $\mathcal{B}$ if every relation of $\mathcal{R}$ is accessible in $(\mathcal{A}, \mathcal{B})$.

REMARK. Mostowski's notion of weak power [12] corresponds to the case $\mathcal{B}=\left\langle S^{*}(B) ; \subseteq, \mathrm{FIN}\right\rangle$, i.e. to the "simplest" index structure.

Theorem $14([6])$. (i) With the above notations, if $\operatorname{Th}(\mathcal{A})$ and $\operatorname{Th}(\mathcal{B})$ are decidable and $\mathcal{C}$ is a generalized weak power of $\mathcal{A}$ relative to $\mathcal{B}$ then the elementary theory of $\mathcal{C}$ is decidable.

(ii) The decision problem for $\operatorname{Th}(\mathcal{B})$ reduces to the one for $\operatorname{Th}\left(S^{+}(B)\right.$; $\left.\subseteq, \mathcal{R}_{B}^{\prime}\right)$ where $\mathcal{R}_{B}^{\prime}$ corresponds to the relations of $\mathcal{R}_{B}$ restricted to $S^{+}(B)$.

In what follows we shall deal with a single class of index structures, namely the structures

$$
I_{\alpha}=\left\langle S^{*}(\alpha) ; \subseteq, \text { FIN },=\text {, SINGL, ISEG }, \prec, \mathrm{U}, \text { EMPTY }\right\rangle
$$

where

- $\alpha$ is an ordinal,

- $I_{\alpha}=\operatorname{SINGL}(X)$ iff $X$ is a singleton; 
- $I_{\alpha}=\operatorname{ISEG}(X)$ iff $X$ is an initial segment of $\alpha$;

- $I_{\alpha}=X \prec Y$ iff (either $X$ or $Y$ is empty, or $X, Y$ are nonempty finite sets and $\operatorname{Sup}(X)<\operatorname{Inf}(Y))$;

- $I_{\alpha}=\mathrm{U}(X, Y, Z)$ iff $X \cup Y=Z$;

- $I_{\alpha}=\operatorname{EMPTY}(X)$ iff $X$ is empty.

Proposition 15. The elementary theory of $I_{\alpha}$ is decidable for every ordinal $\alpha$.

Proof. By Theorem 14(ii) it suffices to show that the theory of

$$
I_{\alpha}^{\prime}=\left\langle S^{+}(\alpha) ; \subseteq,=\text {, SINGL, ISEG }, \prec, \mathrm{U}, \text { EMPTY }\right\rangle
$$

is decidable. Now by Büchi $[1,2]$ the weak monadic second-order theory of $\langle\alpha,<\rangle$ is decidable for every ordinal $\alpha$, and it is not difficult to check that $\operatorname{Th}\left(I_{\alpha}^{\prime}\right)$ is reducible to the latter theory.

We first deal with the theory of $\left\langle\omega^{\xi} ;{ }_{l}\right\rangle$ (for any ordinal $\xi$ ).

Lemma 16. Let $\beta=\sum_{i=1}^{k} \omega^{\beta_{i}} b_{i}>0(C N F)$. An ordinal $\alpha>0$ is a left-hand divisor of $\beta$ if and only if either

(i) $\operatorname{deg}(\alpha)<\beta_{k}$, or

(ii) $\alpha=\omega^{\beta_{j}} c+\sum_{i=j+1}^{k} \omega^{\beta_{i}} b_{i}$ with $c \mid b_{j}$.

Proof. Let us write $\alpha=\omega^{\alpha_{0}} \alpha_{1}$ where $\alpha_{1}$ is a successor ordinal, and let $\beta^{\prime}$ be such that $\beta=\omega^{\beta_{k}} \beta^{\prime}$. One has $\left.\alpha\right|_{l} \beta$ if and only if there exist an ordinal $\gamma_{0}$ and a successor ordinal $\gamma_{1}$ such that

$$
\omega^{\alpha_{0}} \alpha_{1} \omega^{\gamma_{0}} \gamma_{1}=\omega^{\beta_{k}} \beta^{\prime}
$$

If $\gamma_{0} \geq 1$ then (2) is equivalent to

$$
\omega^{\alpha_{0}+\operatorname{deg}\left(\alpha_{1}\right)+\gamma_{0}} \gamma_{1}=\omega^{\beta_{k}} \beta^{\prime},
$$

i.e. to

$$
\omega^{\operatorname{deg}(\alpha)+\gamma_{0}} \gamma_{1}=\omega^{\beta_{k}} \beta^{\prime},
$$

which has a solution if and only if $\operatorname{deg}(\alpha)<\beta_{k}$.

If $\gamma_{0}=0$ then (2) is equivalent to

$$
\omega^{\alpha_{0}} \alpha_{1} \gamma_{1}=\omega^{\beta_{k}} \beta^{\prime},
$$

which has a solution if and only if $\alpha_{0}=\beta_{k}$ and $\left.\alpha_{1}\right|_{l} \beta^{\prime}$. Now from $\beta=\omega^{\beta_{k}} \beta^{\prime}$ it follows that

$$
\beta^{\prime}=b_{k}\left(\omega^{\beta_{k-1}-\beta_{k}}+1\right) b_{k-1}\left(\omega^{\beta_{k-2}-\beta_{k-1}}+1\right) b_{k-2} \ldots\left(\omega^{\beta_{1}-\beta_{2}}+1\right) b_{1},
$$

which, by Proposition 4 , yields that $\alpha_{1}$ must have the form

$$
\alpha_{1}=b_{k}\left(\omega^{\beta_{k-1}-\beta_{k}}+1\right) b_{k-1}\left(\omega^{\beta_{k-2}-\beta_{k-1}}+1\right) b_{k-2} \ldots\left(\omega^{\beta_{j}-\beta_{j+1}}+1\right) c
$$


for some $c<\omega$ which divides $b_{j}$. This together with $\alpha=\omega^{\beta_{k}} \alpha_{1}$ leads to

$$
\alpha=\omega^{\beta_{j}} c+\sum_{i=j+1}^{k} \omega^{\beta_{i}} b_{i} .
$$

Proposition 17. For every ordinal $\xi,\left\langle\omega^{\xi} ;\left.\right|_{l}\right\rangle$ is isomorphic to a weak generalized power of $\langle\omega ; \mid\rangle$ relative to $I_{\xi}$.

Proof. Denote by $N_{\xi}$ the set of functions $f: \xi \rightarrow \omega$ such that $f(\gamma)=0$ for all but finitely many $\gamma$ (i.e. $N_{\xi}=\omega_{0}^{(\xi)}$ with the previous notations). Consider $c: \omega^{\xi} \rightarrow N_{\xi}$ which maps every $\alpha<\omega^{\xi}$ to the function $c(\alpha) \in N_{\xi}$ defined as follows: for every $\gamma<\xi,[c(\alpha)](\gamma)$ is the coefficient of $\omega^{\gamma}$ in the normal form of $\alpha$. The mapping $c$ is $1-1$, thus there exists a binary relation $\operatorname{DIV}_{L}$ on $N_{\xi}$ such that $\left\langle\omega^{\xi} ;\left.\right|_{l}\right\rangle$ and $\left\langle N_{\xi} ; \operatorname{DIV}_{L}\right\rangle$ are isomorphic. Let us show that $\left\langle N_{\xi} ; \mathrm{DIV}_{L}\right\rangle$ is a generalized weak power of $\langle\omega ; \mid\rangle$ relative to $I_{\xi}$. For this one must prove that $\operatorname{DIV}_{L}$ is accessible in $\left(\langle\omega ; \mid\rangle, I_{\xi}\right)$. This is done by translating, via the mapping $c$, the conditions given by the previous lemma. For $f, f^{\prime} \in N_{\xi}$, we have

$$
\left\langle N_{\xi} ; \operatorname{DIV}_{L}\right\rangle \models \operatorname{DIV}_{L}\left(f, f^{\prime}\right)
$$

if and only if

$$
\begin{aligned}
& I_{\xi}= \\
& \neg \operatorname{EMPTY}\left(X_{1}\right) \Rightarrow \\
& \left\{\neg \operatorname{EMPTY}\left(X_{2}\right) \wedge\right. \\
& {\left[X_{2} \prec X_{1} \quad\right\} \operatorname{case}(\mathrm{i})} \\
& \left.\begin{array}{l}
\left(U\left(X_{2}, X_{4}, X_{5}\right) \wedge X_{2} \prec X_{3} \wedge X_{4} \prec X_{5}\right. \\
\left.\left.\left.\wedge \neg \operatorname{EMPTY}\left(X_{5}\right) \Rightarrow \operatorname{SingL}\left(X_{5}\right)\right)\right]\right\}
\end{array}\right\} \text { case (ii) }
\end{aligned}
$$

where

$$
\begin{aligned}
& X_{1}=\left\{\gamma<\xi: f^{\prime}(\gamma) \neq 0\right\} \\
& X_{2}=\{\gamma<\xi: f(\gamma) \neq 0\}, \\
& X_{3}=\left\{\gamma<\xi: f(\gamma)=0 \wedge f^{\prime}(\gamma) \neq 0\right\} \\
& X_{4}=\left\{\gamma<\xi: f(\gamma)=f^{\prime}(\gamma) \wedge f(\gamma) \neq 0\right\}, \\
& X_{5}=\left\{\gamma<\xi: f(\gamma) \mid f^{\prime}(\gamma) \wedge f(\gamma) \neq f^{\prime}(\gamma) \wedge f^{\prime}(\gamma) \neq 0\right\}
\end{aligned}
$$

Moreover one easily finds formulas $F_{1}, \ldots, F_{5}$ in the language $\{\mid\}$ such that $X_{j}=\left\{\gamma<\xi:\langle\omega ; \mid\rangle \models F_{j}\left(f(\gamma), f^{\prime}(\gamma)\right)\right\}$ for $j=1, \ldots, 5$.

Theorem 18. For every ordinal $\xi$, Th $\left\langle\omega^{\xi} ;\left.\right|_{l}\right\rangle$ is decidable.

Proof. By Proposition 15, $\operatorname{Th}\left(I_{\xi}\right)$ is decidable; moreover $\operatorname{Th}(\omega ; \mid)$ is decidable as it is reducible to $\operatorname{Th}(\omega ; \times)$ which is decidable by [12]. Thus by 
Proposition 17 and Theorem 14 we see that $\operatorname{Th}\left(N_{\xi} ; \mathrm{DIV}_{L}\right)$ is decidable, from which the result follows.

We shall now consider the theory of $\left\langle\omega^{\omega^{\xi}} ;\left.\right|_{r}\right\rangle$ for an ordinal $\xi$, and prove that it is decidable. The proof shares some ideas with the previous one for $\left\langle\omega^{\xi} ;\left.\right|_{l}\right\rangle$.

LEMmA 19. Let $\alpha=\omega^{\alpha_{0}} \alpha_{1}, \beta=\omega^{\beta_{0}} \beta_{1}$, where $\alpha_{1}, \beta_{1}$ are successor ordinals. Then $\left.\alpha\right|_{r} \beta$ if and only if one of the following conditions holds:

(i) $\alpha_{0}=0$ and $\left.\alpha_{1}\right|_{r} \beta_{1}$;

(ii) $\alpha_{0}>0, \beta_{0}=\gamma+\alpha_{0}$ for some $\gamma$, and $\alpha_{1}=\beta_{1}$.

Proof. One has $\left.\alpha\right|_{r} \beta$ if and only if

$$
\omega^{\beta_{0}} \beta_{1}=\omega^{\gamma_{0}} \gamma_{1} \omega^{\alpha_{0}} \alpha_{1}
$$

for some ordinal $\gamma_{0}$ and some successor ordinal $\gamma_{1}$.

If $\alpha_{0}=0$ then (3) is equivalent to $\omega^{\beta_{0}} \beta_{1}=\omega^{\gamma_{0}} \gamma_{1} \alpha_{1}$, which by Corollary 3 admits a solution if and only if $\left.\alpha_{1}\right|_{r} \beta_{1}$.

Now if $\alpha_{0}>0$ then (3) is equivalent to $\omega^{\beta_{0}} \beta_{1}=\omega^{\gamma_{0}+\operatorname{deg}\left(\gamma_{1}\right)+\alpha_{0}} \alpha_{1}$, which has a solution if and only if $\beta_{0}=\gamma+\alpha_{0}$ for some $\gamma$, and $\alpha_{1}=\beta_{1}$.

LEMMA 20. For every ordinal $\xi$, the decision problem for $\operatorname{Th}\left(\omega^{\omega^{\xi}} ;\left.\right|_{r}\right)$ reduces to the one for $\operatorname{Th}\left(\left[\omega^{\omega^{\xi}}\right]_{S} ;\left.\right|_{r}\right)$, where $\left[\omega^{\omega^{\xi}}\right]_{S}$ denotes the set of successor ordinals $<\omega^{\omega^{\xi}}$.

Proof. A first observation is that the decision problem for $\operatorname{Th}\left(\omega^{\omega^{\xi}} ;\left.\right|_{r}\right)$ reduces to the one for $\operatorname{Th}\left(\omega^{\omega^{\xi}} \backslash\{0\} ;\left.\right|_{r}\right)$. Indeed using the logical equivalences $\left(\left.\left.0\right|_{r} x \Leftrightarrow 0\right|_{r} 0\right)$ and $\left(\left.x\right|_{r} 0 \Leftrightarrow x=0\right)$, one shows rather easily that any $\left\{\left.\right|_{r}\right\}$-sentence $\theta$ is logically equivalent to a $\left\{\left.\right|_{r}\right\}$-sentence $\theta^{\prime}$ in which all quantifications are relativized to the (definable) set $\omega^{\omega^{\xi}} \backslash\{0\}$. Moreover one can obtain $\theta^{\prime}$ from $\theta$ in a recursive way.

Now observe that the function $\tau: \omega^{\omega^{\xi}} \backslash\{0\} \rightarrow \omega^{\xi} \times\left[\omega^{\omega^{\xi}}\right]_{S}$ which maps any ordinal $\alpha=\omega^{\alpha_{0}} \alpha_{1}>0$, where $\alpha_{1}$ is a successor ordinal, to the couple $\left(\alpha_{0}, \alpha_{1}\right)$, is one-one. This together with the "simple" conditions of the previous lemma allows us to see $\left\langle\omega^{\omega^{\xi}} \backslash\{0\} ;\left.\right|_{r}\right\rangle$ as a product of two structures, in the following sense: let $R_{1}, R_{2}$ be two binary relational symbols, and let $\mathcal{M}_{1}, \mathcal{M}_{2}$ be two $\left\{R_{1}, R_{2}\right\}$-structures with respective domains $\omega^{\xi}$ and $\left[\omega^{\omega^{\xi}}\right]_{S}$, and where $R_{1}, R_{2}$ are interpreted as follows:

- $\mathcal{M}_{1}=R_{1}(x, y)$ if and only if $x=0$,

- $\mathcal{M}_{2} \models R_{1}(x, y)$ if and only if $\left.x\right|_{r} y$,

- $\mathcal{M}_{1}=R_{2}(x, y)$ if and only if there exists $\gamma<\omega^{\xi}$ such that $x+\gamma=y$,

- $\mathcal{M}_{2} \models R_{2}(x, y)$ if and only if $x=y$. 
Let $\mathcal{N}=\mathcal{M}_{1} \times \mathcal{M}_{2}$. It follows from the previous lemma that given any $c_{1}, c_{2} \in|\mathcal{N}|$,

$$
\mathcal{N} \models \bar{R}_{1}\left(c_{1}, c_{2}\right) \vee \bar{R}_{2}\left(c_{1}, c_{2}\right)
$$

if and only if

$$
\left\langle\omega^{\omega^{\xi}} \backslash\{0\} ;\left.\right|_{r}\right\rangle=\left.\tau^{-1}\left(c_{1}\right)\right|_{r} \tau^{-1}\left(c_{2}\right) .
$$

Thus $\operatorname{Th}\left(\omega^{\omega^{\xi}} \backslash\{0\} ;\left.\right|_{r}\right)$ reduces to $\operatorname{Th}(\mathcal{N})$, which is decidable if $\operatorname{Th}\left(\mathcal{M}_{1}\right)$ and $\operatorname{Th}\left(\mathcal{M}_{2}\right)$ are (by [12]). The theory of $\mathcal{M}_{1}$ is an unessential extension of $\operatorname{Th}\left(\omega^{\xi} ;+\right)$ which is decidable by [2], and $\operatorname{Th}\left(\mathcal{M}_{2}\right)$ is interpretable in $\operatorname{Th}\left(\left[\omega^{\omega^{\xi}}\right]_{S} ;\left.\right|_{r}\right)$.

We shall use again the Feferman-Vaught theorem, with the following class of factor structures: for every ordinal $\xi$, set

$$
E_{\xi}=\left\langle\omega^{\xi} \times(\omega \backslash\{0\}) ;={ }^{1}, \mathrm{ZERO}^{1},\left.\right|^{2}, \mathrm{ONE}^{2}\right\rangle
$$

where

- $E_{\xi} \models h={ }^{1} h^{\prime}$ if and only if $\pi_{1}(h)=\pi_{1}\left(h^{\prime}\right)\left(\pi_{i}\right.$ denotes the $i$ th projection),

- $E_{\xi}=\mathrm{ZERO}^{1}(h)$ if and only if $\pi_{1}(h)=0$,

- $\left.E_{\xi} \models h\right|_{2} h^{\prime}$ if and only if $\pi_{2}(h) \mid \pi_{2}\left(h^{\prime}\right)$

- $E_{\xi}=\mathrm{ONE}^{2}(h)$ if and only if $\pi_{2}(h)=1$.

Proposition 21. For every ordinal $\xi, \operatorname{Th}\left(\left[\omega^{\omega^{\xi}}\right]_{S} ;\left.\right|_{r}\right)$ can be interpreted in a weak generalized power of $E_{\xi}$ relative to $I_{\omega}$.

Proof. By Proposition 4 every successor ordinal $\beta<\omega^{\omega^{\xi}}$ can be written in a unique way as

$$
\beta=\left(\omega^{\lambda_{n}}+1\right) b_{n}\left(\omega^{\lambda_{n-1}}+1\right) b_{n-1} \ldots\left(\omega^{\lambda_{0}}+1\right) b_{0}
$$

with $0<b_{i}<\omega(i=0, \ldots, n), \lambda_{n} \geq 0$, and $\lambda_{j}>0$ whenever $j<n$.

Consider the set $M_{\xi}$ of functions from $\omega$ to the set of couples of ordinals in $\omega^{\xi} \times(\omega \backslash\{0\})$ such that $f(n) \neq(0,1)$ for finitely many $n$ (i.e. $M_{\xi}=$ $\left(\omega^{\xi} \times(\omega \backslash\{0\})\right)_{(0,1)}^{(\omega)}$ with our notations). Consider $d:\left[\omega^{\omega^{\xi}}\right]_{S} \rightarrow M_{\xi}$ which maps every $\beta \in\left[\omega^{\omega^{\xi}}\right]_{S}$ to the function $d(\beta)$ defined as follows: if $\beta$ is in the form (4) then we set $[d(\beta)](i)=\left(\lambda_{i}, b_{i}\right)$ for every $i \leq n$ and $[d(\beta)](i)=(0,1)$ whenever $i>n$. The mapping $d$ is not onto, but is injective, thus if we denote by $D_{\xi}$ the range of $d$, there exists a binary relation $\operatorname{DIV}_{R}$ such that $\left\langle\left[\omega^{\omega^{\xi}}\right]_{S} ;\left.\right|_{r}\right\rangle \cong\left\langle D_{\xi} ; \operatorname{DIV}_{R}\right\rangle$. We shall prove that $\left\langle M_{\xi} ; D_{\xi}, \operatorname{DIV}_{R}\right\rangle$ (where $D_{\xi}$ is to be understood as a unary relation) is a weak generalized power of $E_{\xi}$ relative to $I_{\omega}$, which will yield the required result. Note that it is sufficient to show that $\mathrm{DIV}_{R}$ is accessible in $\left(E_{\xi}, I_{\omega}\right)$, since $D_{\xi}$ is definable in $\left\langle M_{\xi} ; \mathrm{DIV}_{R}\right\rangle$.

In order to show that $\operatorname{DIV}_{R}$ is accessible in $\left(E_{\xi}, I_{\omega}\right)$, consider an ordinal $\beta \in\left[\omega^{\omega^{\xi}}\right]_{S}$ written in the form (4). Proposition 4 yields that $\alpha \in\left[\omega^{\omega^{\xi}}\right]_{S}$ is a 
right-hand divisor of $\beta$ if and only if $\alpha$ can be written as

$$
\alpha=c_{j}\left(\omega^{\lambda_{j-1}}+1\right) b_{j-1} \ldots\left(\omega^{\lambda_{0}}+1\right) b_{0}
$$

where $j \leq n$ and $c_{j} \mid b_{j}$. Therefore $\left.\alpha\right|_{r} \beta$ if and only if $d(\alpha)$ and $d(\beta)$ satisfy the following conditions:

There exists $j<\omega$ such that $[d(\alpha)](i)=[d(\beta)](i)$ for every $i<j$, and either

- $[d(\alpha)](k)=(0,1)$ for every $k \geq j$, or

- $[d(\alpha)](j)=(0, a),[d(\beta)](j)=(\lambda, b)$ with $a \mid b$ and $b \neq 1$, and $[d(\alpha)](k)$ $=(0,1)$ whenever $k>j$.

We now translate these conditions into the appropriate language: given $f, f^{\prime}$ in $M_{\xi}$, one has

$$
\left\langle M_{\xi} ; \operatorname{DIV}_{R}\right\rangle \models \operatorname{DIV}_{R}\left(f, f^{\prime}\right)
$$

if and only if

$$
\begin{aligned}
I_{\omega}= & U\left(T_{1}, T_{2}, T_{3}\right) \wedge \operatorname{ISEG}\left(T_{1}\right) \wedge \operatorname{ISEG}\left(T_{3}\right) \wedge\left(\operatorname{SINGL}\left(T_{2}\right) \vee \operatorname{EMPTY}\left(T_{2}\right)\right) \\
& \wedge \mathrm{U}\left(T_{4}, T_{5}, T_{6}\right) \wedge \operatorname{ISEG}\left(T_{4}\right) \wedge \operatorname{ISEG}\left(T_{6}\right) \wedge\left(\operatorname{SINGL}\left(T_{5}\right) \vee \operatorname{EMPTY}\left(T_{5}\right)\right)
\end{aligned}
$$

with

$$
\begin{aligned}
& T_{1}=\left\{i<\omega: f^{\prime}(i)=(a, b) \text { for some } a, b \geq 1\right\}, \\
& T_{2}=\left\{i<\omega: f^{\prime}(i)=(0, a) \text { for some } a \geq 2\right\}, \\
& T_{3}=\left\{i<\omega: f^{\prime}(i) \neq(0,1)\right\}, \\
& T_{4}=\left\{i<\omega: f(i)=f^{\prime}(i) \text { and } f(i) \neq(0,1)\right\}, \\
& T_{5}=\left\{i<\omega: f(i)=(0, b), f^{\prime}(i)=(\lambda, a) \text { with } b \mid a \text { and } b \neq 1\right\}, \\
& T_{6}=\{i<\omega: f(i) \neq(0,1)\} .
\end{aligned}
$$

Moreover one easily finds formulas $F_{1}, \ldots, F_{6}$ in the language of $E_{\xi}$ such that $T_{j}=\left\{i<\omega: E_{\xi} \models F_{j}(g(i))\right\}$ for $j=1, \ldots, 6$.

The first line in the above formula expresses that $f^{\prime} \in D_{\xi}$, and the second one translates the above italic conditions (moreover the whole formula implies $\left.f \in D_{\xi}\right)$.

Proposition 22. The theory of $E_{\xi}$ is decidable.

Proof. One can use the same trick as in Lemma 20 and show that $\operatorname{Th}\left(E_{\xi}\right)$ is interpretable in the product of two structures with respective domains $\omega^{\xi}$ and $\omega \backslash\{0\}$. This allows us to reduce the problem to showing decidability of $\operatorname{Th}\left(\omega^{\xi} ;=, 0\right)$ and $\operatorname{Th}(\omega \backslash 0 ; \mid, 1)$. The first theory is, apart from the trivial case $\xi=0$, essentially the theory of equality over an infinite set, which is decidable, and the second one reduces to $\operatorname{Th}(\omega ; \times)$, which is again decidable by [12]. 
From the previous results and Theorem 14 we finally deduce:

Theorem 23. For every ordinal $\xi, \operatorname{Th}\left\langle\omega^{\omega^{\xi}} ;\left.\right|_{r}\right\rangle$ is decidable.

5. Conclusion. We shall conclude with two questions related to the previous results.

First of all, while the spirit of the present paper is rather "syntactic", it would be interesting to exhibit convenient axiom systems for the previous theories.

Another interesting related problem has to do with multiplicative equations over ordinals. An example is the equation $x y=y x$ which was studied by Sierpiński [17] (he proved that the solutions are: either $x, y<\omega$, or $x^{n}=y^{m}$ for some $\left.m, n<\omega\right)$. Is it decidable whether a finite system of multiplicative equations (with constants) has a nontrivial solution?

\section{References}

[1] J. R. Büchi, Weak second-order arithmetic and finite automata, Z. Math. Logik Grundlagen Math. 6 (1960), 66-92.

[2] - , Transfinite automata recursions and weak second order theory of ordinals, in: Logic Methodology Philos. Sci. (Proc. 1964 Internat. Congr.), North-Holland, 1965, $3-23$.

[3] P. Cegielski, Théorie élémentaire de la multiplication des entiers naturels, in: Model Theory and Arithmetic (Paris, 1979/80), Lecture Notes in Math. 890, Springer, 1981, 44-89.

[4] J. E. Doner, A. Mostowski and A. Tarski, The elementary theory of well-ordering - a metamathematical study, in: Proc. Logic Colloquium '77, Stud. Logic Found. Math. 96, North-Holland, 1978, 1-54.

[5] A. Ehrenfeucht, An application of games to the completeness problem for formalized theories, Fund. Math. 49 (1961), 129-141.

[6] S. Feferman and R. L. Vaught, The first order properties of products of algebraic systems, ibid. 47 (1959), 57-103.

[7] B. R. Hodgson, On direct products of automaton decidable theories, Theoret. Comput. Sci. 19 (1982), 331-335.

[8] J. E. Hopcroft and J. D. Ullman, Introduction to Automata Theory, Languages, and Computation, Addison-Wesley, 1979.

[9] E. Jacobsthal, Über der Aufbau der transfiniter Arithmetik, Math. Ann. 66 (1909), 145-194.

[10] F. Maurin, Ehrenfeucht games and ordinal addition, Ann. Pure Appl. Logic 89 (1997), 53-73.

[11] M. L. Minsky, Recursive unsolvability of Post's problem of "tag" and other topics in theory of Turing machines, Ann. of Math. 74 (1961), 437-455.

[12] A. Mostowski, On direct products of theories, J. Symbolic Logic 17 (1952), 1-31.

[13] A. Mostowski and A. Tarski, Arithmetical classes and types of well ordered systems, Bull. Amer. Math. Soc. 55 (1949), p. 65. 
[14] M. Presburger, On the completeness of a certain system of arithmetic of whole numbers in which addition occurs as the only operation (transl. by D. Jacquette), Hist. Philos. Logic 12 (1991), 225-233.

[15] W. J. Quine, Concatenation as a basis for arithmetic, J. Symbolic Logic 11 (1946), 105-114.

[16] M. O. Rabin, Decidable theories, in: Handbook of Mathematical Logic, J. Barwise (ed.), Stud. Logic Found. Math. 90, North-Holland, 1977, 595-629.

[17] W. Sierpiński, Cardinal and Ordinal Numbers, 2nd ed., PWN, Warszawa, 1965.

[18] T. Skolem, Über gewisse Satzfunktionen in der Arithmetik, Skr. Norske VidenskapsAkademie i Oslo 7 (1930).

[19] R. L. Vaught, On the work of Andrzej Ehrenfeucht in model theory, in: Structures in Logic and Computer Science. A Selection of Essays in Honor of Andrzej Ehrenfeucht (J. Mycielski, ed.), Lecture Notes in Comput. Sci. 1261, Springer, 1997, 1-13.

LACL, Département d'Informatique

Faculté des Sciences et Technologie

61 avenue du Général de Gaulle

94010 Créteil Cedex, France

E-mail: bes@logique.jussieu.fr

Received 28 April 1999;

in revised form 20 April 2001 